\title{
Analysis on the Practice of Virtual Reality Technology in Electric Emergency Repair Practice
}

\author{
Huang Chengyun, Guo Jianlong, Xiong Shan, Hao Tengfei \\ Guangzhou Guangdong, 510520, China
}

Keywords: Virtual reality technology; electric power; emergency repair drill; practice

\begin{abstract}
China has a vast territory and a wide distribution network, which has many factors affecting the stability and safety of the distribution network. In recent years, China's emphasis on the quality of distribution network operation has been continuously improved, and research on power emergency management has been strengthened, and emergency work systems such as emergency organization and emergency materials reserve have been formulated. This paper analyzes the technical architecture of power emergency simulation system and the functional architecture and design of power emergency simulation system, and studies the practice of virtual reality technology in power emergency repair drill.

China's power grids are large in scale and complex in structure. These power grids are exposed to the air for a long time and are highly susceptible to the external environment. In order to ensure the normal electricity demand of residents and enterprises, if there is a power outage, the power company needs to repair it in time and resume power supply as soon as possible. The use of virtual reality technology to establish a power emergency drill simulation system can enhance the understanding and mastery of commanders and rescuers on emergency work processes and job responsibilities during the drill process, and effectively improve the coordination effect of various departments.
\end{abstract}

\section{The meaning and features of virtual reality technology}

\subsection{Virtual reality meaning}

Virtual reality technology belongs to the development direction of simulation technology. It is a new technology formed by simulation technology, computer graphics discipline, human-machine structure technology, multimedia technology, sensor technology and other technologies. Virtual reality technology belongs to cross-technology. Virtual reality technology mainly includes content such as simulation environment, perception, and natural skills. The real-time, dynamic three-dimensional graphics are formed by the computer, so that the simulation environment can be achieved. Virtual reality technology needs to have the perceptual capabilities that humans need. It not only includes the visual perception formed by computer graphics technology, but also needs to be aware of touch, motion, smell, taste and so on. Natural skills refer to human head rotation, eye rotation, gestures, etc., and the computer analyzes the participant's motion data, responds to user input commands in a timely manner, and feeds them back to the user. The sensing device mainly refers to a three-dimensional interactive device.

\subsection{Virtual reality technology features}

Virtual reality technology mainly includes the following characteristics: First, conceived. This feature is mainly manifested in the broad imagination space of virtual reality technology. Using virtual reality technology can not only simulate the real environment, but also expand human thinking, set up a different environment from actual life through subjective conjecture, and perform simulation analysis; secondly, mutuality. This feature mainly means that the user can manipulate the objects in the virtual environment, and at the same time, the corresponding feedback can be obtained from the virtual environment, for example, the power economy repair drill system built 
using virtual reality technology, during the simulation process, the maintenance personnel can reach out and operate the virtual repair equipment. At this point, the repair personnel can feel the maintenance equipment, and can feel the weight of the maintenance equipment, and the maintenance equipment will also move and operate along with the maintenance personnel; finally, immersion. It means that the user will operate as the protagonist during the simulation. The user can obtain the same olfactory, auditory, visual, and tactile experience as the real environment in the simulated environment.

\section{Power emergency simulation system construction goals and principles}

\subsection{Electricity emergency simulation system composition and construction process}

The power emergency simulation system is mainly composed of hardware system, software system and database system. The hardware system is mainly composed of a computer, a sound image processing system, a sensing system, etc.; the software system is mainly composed of a three-dimensional modeling software and a scene texture generating software; the database system mainly includes a three-dimensional environment model database, an environmental texture image database, and the like. When constructing a virtual screen, you need to use the 3DMax modeling tool to build a 3D model based on the actual situation and environment to be simulated. After that, you need to output and save the set amount of animation and character model. Finally, you need to import the saved files into Vir-tools and set the corresponding scripts to ensure that the entire emergency simulation system can be used normally.

\subsection{Application of Virtual Reality Technology in Power Emergency Simulation System}

\subsubsection{Virtual scene interaction technology}

When interactively simulating a scene or a role, it is necessary to pay attention to its synergy, and the entire interactive operation can be completed according to the environment and three-dimensional operation, thereby creating a good emergency drill atmosphere.

\subsubsection{Multiplayer online technology}

By configuring an efficient network linkage system, the online connection function can be realized, and the database can be collated and processed to realize the goal of multi-person connection.

\subsubsection{Collision detection algorithm}

In the process of simulation, it is necessary to scientifically select the appropriate collision detection algorithm to analyze whether the simulation environment is real and the motion of the object is realistic. At the same time, it is necessary to check whether the collision environment is reasonable according to the positional relationship between the character and the building.

\subsubsection{Particle effect technology}

In order to achieve the goal of simulating the real environment, you can use the particle effect 3D technology to simulate the actual environment, which can effectively improve the simulation speed and quality.

\subsubsection{Fusion 3D technology}

In the whole simulation process, it is necessary to scientifically select the analysis methods such as submergence analysis, geological analysis and sunshine analysis to simulate and analyze multi-dimensional space, and also need to integrate 3D simulation technology in the analysis process. This technology can be used to simulate data related to circuit equipment and improve the authenticity of emergency drills. 


\section{Power emergency simulation system technical architecture}

In the construction of the electric power emergency simulation system, the architecture model combining Client/Server and Browser/Server is selected for research. The study found that the Client/Server structure database operation function and transaction processing function are strong. Therefore, the use of this structural mode can effectively ensure the security and integrity of the data information, while ensuring the security and integrity of the entire system technology in the emergency processing simulation process, and the related operations are also simpler. If you need to develop and build a large-scale system, you can use 3D virtual simulation system technology to process massive data information. In addition, it can improve the stability and security of network communication and ensure the stable operation of the client design interface. In the process of power emergency repair and rehearsal, the J2EE multi-layer architecture design background module is used. This architecture can ensure good interactivity and coupling between different modules, thus improving software utilization. After unified research and use, it can improve the software and hardware operation effects of the power emergency simulation system, and make full use of the network equipment, security equipment and servers of the power emergency simulation system. In addition, the entire power emergency repair drill process requires data support in the support layer and application layer.

\section{Functional architecture and design of power emergency simulation system}

\subsection{Power emergency simulation system functional architecture}

The virtual reality technology can provide technical support for the power emergency simulation system. The power emergency simulation system mainly includes the modules of the drill configuration management module, the drill execution module, the single skill module, and the drill evaluation module. The drill configuration management module has the function of maintaining data; the single skill module can be used to establish a contact and assessment system.

\subsection{Functional design of power emergency simulation system}

\subsubsection{Drill configuration management module}

The entire drill configuration management module includes four sub-modules (drill event management, participation information management, drill scenario task configuration, and drill script configuration). The main function of the drill event management module is to set basic information related to the drill, the location of the fault location, etc. The main function of the participation information management module is to manage the participating participants, tasks, drill scenarios, etc. The main function of the drill scenario task configuration module is manage drill scenarios and tasks, and set drill scenarios and drill tasks according to the work departments of the participating drills; the main function of the drill script configuration module is to send fault video, pictures and other information to the participating participants according to the script at the set time.

\subsubsection{Drill execution module}

The drill execution module is composed of three sub-modules: a rights management module, an information interaction module, and an emergency response module. The rights management module mainly manages user login, manager monitoring and other rights; the information interaction module mainly needs to exchange information such as announcement, information and call; the emergency response module has common functions and business functions, wherein the common functions mainly include viewing the map and transforming the scene. And other functions, while business functions mainly include functions such as response initiation and response actions. The exercise execution module will affect the normal operation and use of the entire power emergency simulation system. Therefore, in the design and construction process, the design and construction of the exercise execution module should be emphasized. Since the electric power 
emergency simulation system needs to set different drill scenarios and drill tasks according to the departments participating in the drill, and strictly control the authority of the users and managers, it is necessary to ensure that the exercise execution system information interaction function is good, so as to ensure that the entire drill can be smoothly performed.

\subsubsection{Single skill module}

Different from the drill execution module, the single skill module pays more attention to whether the emergency repair personnel can master the repair knowledge and repair skills in different environments, and also pays attention to the assessment of the professional skills of the emergency repair personnel. In the single skill module, need to set the function of adding, deleting, and modifying etc. so that users can query their skills training and assessment results. By constructing a single skill function in the current emergency drill simulation system, the training can be targeted and effective, and the professional skill level of the participating participants can be improved through training. This is of great significance to improving the professional level of emergency repair personnel and ensuring the stability of the distribution network.

\subsubsection{Drill evaluation module}

After the drill is completed, the drill activity needs to be evaluated. The expert team will evaluate the drill effect based on the evaluation template. The drill evaluation module automatic evaluation function can form an evaluation report based on the drill data. After the emergency drill is completed, the power emergency simulation system drill evaluation module can evaluate the entire drill activity, and at the same time evaluate the professional skills and comprehensive ability level of the participating drill personnel, thus stimulating the enthusiasm of the relevant personnel to participate in the drill.

\section{Practice of virtual reality technology in power emergency repair drill}

\subsection{Case overview}

Taking a power emergency repair drill of a $10 \mathrm{kV}$ substation as a case study, this substation is to improve the professional ability and professional quality of the repair personnel. After the introduction of virtual reality technology, employees were given emergency repair drills. The drill was set up as a "lightning strike failure" in the substation, and virtual reality technology was used to build a virtual environment with the same substation and line. Afterwards, the drills for receiving emergency repair signals, rushing to the scene of the accident, on-site investigation, filling in emergency repair orders, preparing emergency repair plans, equipped with emergency repair materials, and emergency repairs were conducted. The emergency repair drill involved staff members such as security personnel, repair personnel, and operational personnel. The emergency rescue drills were completed by a number of departments.

\subsection{Analysis of the practical points of virtual reality technology in electric emergency repair drill}

\subsubsection{Realize human-computer interaction}

After the virtual three-dimensional scene is constructed, the electric power repair personnel need to enter the scene to perform the drill, in which the real person, object or virtual technology can be used to set the person, object or device in the virtual scene. The operation scenarios and operating procedures of different types of work are different. Therefore, it is necessary to set up a corresponding action database in the electric power emergency simulation system according to the duties and working environment of the staff, and then the staff participating in the exercise will perform the drill in the simulation scenario. In the process, not only practical operations but also related questions need to be answered. Through this simulation, the staff can grasp the problems that the electric emergency emergency repair work needs to face, which is of great significance to improve the professional skill level of the staff and shorten the power failure time. 


\subsubsection{Building mathematical model}

The electric power emergency simulation system can simulate the collision problem that is very easy to appear in real life. The three-dimensional technology can be used to simulate the corresponding scene. At the same time, the three-dimensional technology can be used to simulate the actual object, but the characteristics and attributes of the object cannot be simulated. Since the grid equipment has its own specific performance and functions, it is necessary to use mathematical models to simulate the characteristics and attributes of the grid equipment, so as to ensure the authenticity and fidelity of the power emergency repair drill. For example, during the operation of the power equipment, it is necessary to maintain a safe distance from the live equipment, and this feature should also be demonstrated during the simulation exercise. The safety distance between electric power workers and live equipment can be known through the Electrical Safety Work Regulations.

In the electric emergency repair drill, if the repair personnel and staff members do not maintain a safe distance from the live equipment, the relevant system will carry out relevant reactions according to the relevant setting system, and prompt the staff to have a risk of electric shock in front of them. In the evaluation process after the drill is completed, the scores of the participants participating in the drills shall be deducted. If the cleaning is more serious, it will be judged that the operation of the drill is incorrect and will not participate in the subsequent drill.

\subsubsection{Building a virtual 3D scene}

In the process of power emergency repair drill, a virtual three-dimensional scene needs to be constructed before simulation can be performed. In this scenario, all power devices must be included to ensure that the simulated scene is the same as the actual scene in life. Therefore, in the construction of virtual three-dimensional scenes, it is necessary to analyze the substation, power equipment, power plant and other factors in detail, and on the basis of ensuring the authenticity of the virtual three-dimensional scene, the rationality and effectiveness of the electric emergency repair drill can be guaranteed. It takes a certain amount of time and money to set up a three-dimensional scene. However, by constructing a virtual three-dimensional scene, the effectiveness of the electric emergency repair drill can be ensured, and all the repair personnel can participate in the electric emergency repair drill work to improve their professional quality and professional ability.

\subsubsection{Predictive reasoning}

When one or more preconditions or judgments are known, the case reasoning can be performed and relevant conclusions can be obtained. Under normal circumstances, in the process of power emergency repair drills, the artificial intelligence technology is used for reasoning work. Because the characteristics and functions of different devices are different, the operation behavior of the staff will affect the repair work. If it is not reasoned, it will not fully reflect the power failure. The electric power emergency repair drill is a drill plan based on the accidents existing in actual life. The general inference engine contains a plurality of events, so when judging whether the power system is faulty, it is necessary to judge whether it has a fault condition, and then it can be judged whether the power system may malfunction.

\section{Conclusion}

In short, the use of virtual reality technology can not only fully demonstrate different power failures, but also show the consequences and damage caused by different power failures to the user, and drill through the three-dimensional scene. Through the electric power emergency repair drill, the professional skill level and adaptability of the repair personnel can be improved, the power system failure time can be shortened, and the economic benefits and social benefits of the power enterprise can be improved. 


\section{Acknowledgements}

Virtual Reality Electric Inspection Interactive Experience System Construction Project

\section{References}

[1] Jialin Gang. Application of Virtual Reality Technology in Electric Power Training[J]. Science and technology innovation report, 2016,13(23):102-102.

[2] Dongdong Wang. Power Safety Supervision Simulation Training System Based on Virtual Reality Technology[J]. Electronic Technology and Software Engineering, 2016(24):49-49.

[3] Xiaolin Diao. Application of Virtual Reality Technology in Electric Energy Measurement Training[J]. Electromechanical engineering technology, 2017(3):111-115.

[4] Yang Su, Bing Zhao. Conception of Building Information Model and Virtual Reality Technology Applied in Power Pipe Gallery[J]. Electric power information and communication technology, 2017(4):26-29.

[5] Hao Chen, Longchuan Yan, Xinpeng Chen,et al. Virtual reality technology and its application in the power industry[J]. Electric power information and communication technology, 2017(5):16-21.

[6] Yiting Cai, Mengxian Chen, Wenbo Dai, et al. Research and Practical Value of Virtual Reality Technology in Power System Training[J]. Electric power information and communication technology, 2017(5):27-32. 